\title{
DETERMINACIÓN DE PATRONES DE VARIACIÓN MORFOLÓGICA DEL GÉNERO MINTHOSTACHYS EN UNCHUS Y CAJATAMBO, MEDIANTE MÉTODOS ESTADÍSTICOS MULTIVARIANTES DE REDUCCIÓN DE DATOS
}

\author{
Doris Gómez Ticerán ${ }^{1}$ \& Olga Solano Dávila ${ }^{1}$ \& Joaquina Albán ${ }^{2}$ \\ Camilo Vásquez ${ }^{2}$ \& Jaime Vásquez ${ }^{1}$ \& Yakov M. Quinteros ${ }^{2}$
}

Resumen.- El estudio fue realizado en la localidad de Unchus y en el distrito de Cajatambo en los departamentos de Ancash y Lima respectivamente. Ambos lugares se encuentran en territorio alto andino. El objetivo del estudio fue realizar un análisis comparativo de la caracterización del género Minthostachys (muña), a partir de muestras colectadas durante los años 2004 y 2005, mediante representaciones gráficas de las variables originales pertenecientes al espacio $\mathbb{R}^{5}$ en $\mathbb{R}^{2}$, usando el Análisis Factorial (Johnson, 2000; Seber, 1984 y otros), Análisis de Componentes Principales y Análisis de Componentes Principales Generalizadas (Flury, 1983; De Paz y Gómez, 2002). Los estudios de las variables morfológicas fueron realizados en el Departamento de Etnobotánica y Botánica Económica del Museo de Historia Natural y la determinación de las especies se realizó en el Herbario USM utilizando el Sistema de Clasificación de Cronquist. Los gráficos realizados usando las variables generadas mediante el análisis de Componentes Principales y Análisis de Componentes Principales Generalizadas, permiten identificar tres especies diferentes de Minthostachys en las comunidades de Unchus y Cajatambo. Se confirman los resultados mediante la $T^{2}$ de Hotelling.

Palabras claves: Análisis de Componentes Principales, Análisis de Componentes Principales Generalizadas, $T^{2}$ de Hotelling, Minthostachys, Cajatambo, Unchus.

\begin{abstract}
The study was conducted in the town of Unchus and in the district of Cajatambo in the departments of Ancash and Lima respectively. Both places are in high Andean territory. The objective of this study was to conduct a comparative analysis of the characterization of gender Minthostachys (muña), from samples collected during the years 2004 and 2005, through graphic representations of the original variables belonging to space $\mathbb{R}^{5}$ to $\mathbb{R}^{2}$ using Analysis
\end{abstract}

\footnotetext{
${ }^{1}$ UNMSMS, Facultad de Ciencias Matemáticas, Lima - Perú

${ }^{2}$ UNMSM, Departamento de Etnobotánica y Botánica Económica - Museo de Historia Natural, Lima - Perú
} 
Factorial (Johnson, 2000; Seber, 1984 et al.), Principal Component Analysis and Principal Component Analysis Generalised (Flury, 1983; De Paz and Gómez, 2002). The morphologyc variables studies were conducted in the Department of Ethnobotany and conomic Botany of the Natural History Museum and the identification of species was conducted in the Herbarium USM using the Classification System Cronquist. The graphics using variables generated by the principal component analysis and Principal Component Analysis Generalised, allow to identify three different species of Minthostachys in communities of Unchus and Cajatambo. The results are confirmed by $T^{2}$ de Hotelling.

Key words: Principal Component Analysis, Principal Component Analysis Generalised, $T^{2}$ Hotelling, Minthostachys, Cajatambo, Unchus.

\section{INTRODUCCION}

Desde 1985, Albán y un grupo de investigadores del Departamento de Etnobotánica y Botánica Económica del Museo de Historia Natural de la UNMSM se han interesado en realizar estudios de poblaciones de plantas medicinales andinas, en particular del género Minthostachys, una de las especies medicinales andinas más fascinantes por sus múltiples usos.

El género Minthostachys se caracteriza por encontrársele entre los 500 y 4000 msnm. Es una planta herbácea cuando joven o subarbustiva cuando adulta, puede medir de 1 a 1.5 metros de alto; es aromática y perenne, de hojas verdes, pecioladas, elípticas-lanceoladas Asimismo se encuentra cubierta de pelos blandos o tricomas (pelos muy cortos, blandos y sensibles al tacto). Los tallos son cuadrangulares y se presentan ampliamente extendidos; erguidos cuando juveniles, y luego se tornan decumbentes (en la madurez), curvados y extendidos en forma irregular. Poseen inflorescencias con muchas flores pendiendo de los tallos. Usualmente crece en bordes de chacras de cultivos, bordes de acequias o sobre terrenos húmedos.

Minthostachys es utilizada por el poblador andino desde tiempos inmemoriales en el tratamiento de la parasitosis, antiespasmódico, con fines alimenticios, para la conservación de alimentos, como repelente de insectos, entre otros. Es así que no sólo contribuye al mejoramiento de la salud del campesino, sino también se manifiesta como recurso valioso y con un gran potencial para la exportación; el cual debe ser aprovechado de una manera sostenible. 
En el cuadro 1 se muestra el catálogo de las Angiospermas y Gimnospermas del Perú (Brako \& Zarucchi, 1993), muestra la distribución del género Minthostachys para el Perú.

\begin{tabular}{|c|c|c|}
\hline ESPECIE & $\begin{array}{l}\text { ALTITUD } \\
\text { (m.s.n.m.) }\end{array}$ & DISTRIBUCIÓN \\
\hline $\begin{array}{c}\text { Minthostachys glabrescens } \\
\text { (Bentham) }\end{array}$ & $2500-4000$ & $\begin{array}{l}\text { Apurímac, Cajamarca, Cuzco, } \\
\text { Junín. }\end{array}$ \\
\hline $\begin{array}{l}\text { Minthostachys mollis } \\
\text { (Grisebach) }\end{array}$ & $500-3500$ & $\begin{array}{l}\text { Amazonas, Arequipa, Cajamarca, Huánuco, } \\
\text { Cuzco, La Libertad, Junín, Lima, Piura. }\end{array}$ \\
\hline $\begin{array}{l}\text { Minthostachys setosa } \\
\text { (Briquet) Epling }\end{array}$ & $1000-1500$ & Puno \\
\hline $\begin{array}{c}\text { Minthostachys tomentosa } \\
\text { (Bentham) }\end{array}$ & $2000-3500$ & $\begin{array}{l}\text { Amazonas, Cajamarca, Cuzco, Huánuco, } \\
\text { Junín, Lima, La Libertad, Ancash. }\end{array}$ \\
\hline $\begin{array}{l}\text { Minthostachys andina } \\
\text { (Britton) Epling }\end{array}$ & $2000-2500$ & Cuzco \\
\hline $\begin{array}{l}\text { Minthostachys mandoniana } \\
\text { (Briquet) Epling }\end{array}$ & $1000-1500$ & Ayacucho \\
\hline $\begin{array}{c}\text { Minthostachys salicifolia } \\
\text { Epling }\end{array}$ & $2500-3000$ & Ayacucho \\
\hline
\end{tabular}

Tabla 1: Minthostachys en el Perú.

Cabe indicar que en el mundo de hoy existe una mayor demanda por productos naturales por lo que es necesario el planteamiento de políticas que alienten el desarrollo de dichos productos en las comunidades andinas.

Hasta el año 2004 no se disponía de registros de Minthostachys en las localidades de Unchus y Cajatambo, por lo que en el año 2004 se realizó la investigación en el distrito de Cajatambo (Gómez et al, 2007) y en el siguiente año se completaron los registros de Minthostachys de la localidad de Unchus.

En el contexto descrito, el objetivo del presente estudio fue identificar las posibles especies de Minthostachys colectadas en el distrito de Cajatambo del Departamento de Lima y de la localidad de Unchus del Departamento de Ancash, mediante métodos estadísticos multivariantes (Manly, 1986; Johnson, 2000; Seber, 1984).

Para tal propósito, se hizo la correspondiente reducción de dimensión mediante el análisis de Componentes Principales Generalizados (Flury, 1983; Gómez, 2002) y se compararon con los resultados obtenidos mediante el análisis clásico a través del análisis de Componentes Principales y el análisis Factorial (Manly B., 2004, entre otros). 


\section{DISEÑO EXPERIMENTAL}

\section{ÁREA DE ESTUDIO}

El distrito de Cajatambo se ubica en la Provincia de Cajatambo, región Lima, en la parte occidental de la cordillera andina, entre el departamento de Ancash y las provincias limeñas de Oyón y Huaura, a una altura de 3,376 m.s.n.m. y tiene una población de aproximadamente 9618 habitantes (INEI,2005) de la que el 56\% es rural. En el área de estudio se encontraron varios pisos climáticos, influenciados principalmente por la altitud y la latitud. En Cajatambo se realizaron muestreos en dos localidades: Cruzjirca y Rancas. En Cruzjirca el clima durante el día es semiseco y frío entre los 3000 y 3200 m.s.n.m., pero durante las noches es frío. En Rancas, que se encuentra aproximadamente entre los 3500 y 4000 m.s.n.m. el clima se vuelve semihúmeḍo y semifrío durante el día, pudiendo llegar incluso a temperaturas bajo cero durante la noche. En Rancas sólo se realizaron muestreos hasta los 3600 m.s.n.m. (límite de distribución actitudinal del genero Minthostachys).

Unchus, localidad cercana a la provincia de Huaraz, se encuentra en el departamento de Ancash aproximadamente entre 3100 y $3200 \mathrm{msnm}$. Su clima es templado durante el día, pero no llega a ser extremadamente frío cuando comparado con las noches cajatambinas.

El muestreo preliminar se realizó entre los meses de Enero a Junio del año 2004, en un gradiente altitudinal de 2800 a 3600 m.s.n.m. en las localidades de Rancas y Cruzjirca en el distrito de Cajatambo; mientras que el muestreo en Unchus se realizó en el mismo periodo del año 2005 en un gradiente de 3050 a 3200 m.s.n.m. Cabe resaltar que los muestreos se realizaron durante las épocas de lluvias (enero - marzo) y durante algunos meses de sequía (abril - junio).

Entre julio y agosto de ambos años se colectaron las muestras botánicas definitivas de Minthostachys, tanto en Cajatambo como en Unchus, las que fueron determinadas taxonómicamente en el Herbario USM, mediante el Sistema de Clasificación de Cronquist.

Las muestras colectadas han sido inventariadas en el Herbario del Museo de Historia Natural y son de propiedad del Departamento de Etnobotánica y Botánica Económica, en cuyas instalaciones se realizaron los estudios taxonómicos y sistemáticos de las muestras.

Las variables morfológicas evaluadas en la rama principal de cada Minthostachys de las muestras recolectadas en Cajatambo y Unchus fueron las siguientes: largo del pedicelo 
de la flor, ancho del pedicelo de la flor, número de venas del cáliz, largo de la corola y ancho de la corola.

\section{METODOLOGÍA ESTADÍSTICA PARA EL ANÁLISIS DE DATOS}

El Análisis de Componentes Principales (ACP) fue propuesto en 1901 por el fundador de la bioestadística, el prominente matemático Karl Pearson, como una solución a algunos problemas que interesaron a los biometristas de aquella época. El ACP ( Seber, 1984; Manly, 2004 ) es un método multivariante de análisis de datos cuyo objetivo fundamental es la reducción de datos de alta dimensión $\mathbb{R}^{p}(p>2)$ en un conjunto de menor dimensión, por ejemplo $\mathbb{R}^{2}$, conservando la máxima variabilidad del problema original, donde las representaciones gráficas y las interpretaciones de las relaciones entre las variables es mucho más sencilla, cuando se disponen de datos correspondientes a una única población.

A partir del vector aleatorio multivariante $\overrightarrow{X^{\prime}}=\left(X_{1}, X_{2}, \ldots, X_{p}\right)$ de $p$ componentes, con vector de medias $\overrightarrow{\mu^{\prime}}=\left(u_{1}, u_{2}, \ldots, u_{p}\right)$ y matriz de covarianzas $\Sigma\left(\sigma_{i j}\right) ; i, j=1, \ldots, p$ se realizan transformaciones que permiten la obtención de combinaciones lineales no correlacionadas y con varianzas decrecientes, $Y_{i}=\beta_{i}^{\prime} \vec{X}, Y_{1}, Y_{2} \ldots, Y_{p}$, denominadas Componentes Principales (CP). Es decir: $\operatorname{Var}\left(Y_{i}\right)=\lambda_{i}, \operatorname{Var}\left(Y_{1}\right) \geq \operatorname{Var}\left(Y_{2}\right) \geq \ldots \geq \operatorname{Var}\left(Y_{p}\right)$, y $\operatorname{Cov}\left(Y_{i}, Y_{j}\right)=0 ; i \neq j, i, j=1, \ldots, p$, donde $\lambda_{i}$ es el i-ésimo autovalor asociado al i-ésimo autovector $\vec{\beta}_{i}=\left(\beta_{i 1}, \ldots, \beta_{i p}\right)$ de la matriz de covarianzas $\Sigma$. Se seleccionan las CP que retienen la máxima variabilidad contenida en las variables originales. Comúnmente en las aplicaciones, donde el objetivo es visualizar los datos en una dimensión bidimensional, $\mathbb{R}^{2}$, se escogen la primera y segunda $\mathrm{CP}$ que en su forma vectorial tienen la siguiente presentación:

$$
\begin{gathered}
\vec{Y}=\left[\begin{array}{l}
Y_{1} \\
Y_{2}
\end{array}\right]=\left[\begin{array}{c}
\overrightarrow{\beta_{1}^{\prime}} \vec{X} \\
\overrightarrow{\beta_{2}^{\prime}} \vec{X}
\end{array}\right] \text { cuyo vector de medias es } \vec{\mu}=\left[\begin{array}{l}
u_{1} \\
u_{2}
\end{array}\right] \\
\text { y matriz de covarianzas } \Sigma_{\vec{Y}}=\left[\begin{array}{cc}
\lambda_{1} & 0 \\
0 & \lambda_{2}
\end{array}\right]
\end{gathered}
$$

Este vector capta la máxima variabilidad de todas las variables originales.

La idea de ACP en un grupo, fue generalizada por Flury a dos grupos o a dos pobla- 
ciones y lo presentó con el nombre de Análisis de Componentes Principales Generalizados $(\mathrm{CPG})$, resultado que a continuación se describe brevemente.

Para los vectores aleatorios $\vec{X}^{(g)}, p$ variantes en cada uno de las poblaciones $g=1,2$, con vectores de medias $\vec{\mu}^{(g)}$ y matrices de covarianzas simétricas definidas positivas $\Sigma_{g}$; con el vector $\vec{a}$ de $p$ componentes, se definen las combinaciones lineales, $Y^{(g)}=\vec{a}^{\prime} \vec{X}^{(g)}$, cuyas varianzas son $\overrightarrow{a^{\prime}} \Sigma_{g} \vec{a}$. Obtener las combinaciones lineales con mayor cociente de varianzas, CPG, $H_{1}(\vec{a})=\frac{\overrightarrow{a^{\prime}} \Sigma_{2} \vec{a}}{\overrightarrow{a^{\prime} \Sigma_{1} \vec{a}}}$ O $H_{2}(\vec{a})=\frac{\overrightarrow{a^{\prime}} \Sigma_{1} \vec{a}}{\overrightarrow{a^{\prime} \Sigma_{2} \vec{a}}}$ para todo vector $\vec{a}$, conduce a la obtención de los autovalores y autovectores de las matrices $\Sigma_{1}^{-1} \Sigma_{2} \circ \Sigma_{2}^{-1} \Sigma_{1}$ respectivamente. Cabe indicar que para aplicar el método, las matrices de covarianzas de las dos poblaciones tienen que ser diferentes. Basado en el principio de unión intersección ( Roy, 1957), la idea de ACP en un grupo y usando los autovectores, $\vec{\beta}_{i}$, de la matriz $\Sigma_{1}^{-1} \Sigma_{2}, i=1, \ldots, p$. Flury (1983) definió variables no correlacionadas en ambos poblaciones, a las que denominó Componentes Principles Generalizados (CPG):

$$
Y_{i}^{(1)}={\overrightarrow{\beta_{i}^{\prime}}}^{\left({ }^{(1)}\right.} \text { y } Y_{i}^{(2)}={\overrightarrow{\beta_{i}^{\prime}}}^{\left({ }^{(2)}\right.}
$$

con varianzas: $\operatorname{Var}\left(Y_{i}^{(1)}\right)=\overrightarrow{\beta_{i}^{\prime}} \Sigma_{1} \vec{\beta}_{i}=1, \operatorname{Var}\left(Y_{i}^{(2)}\right)=\overrightarrow{\beta_{i}^{\prime} \Sigma_{2}} \vec{\beta}_{i}=\lambda_{i}$

y con covarianzas: $\operatorname{cov}\left(Y_{i}^{(1)}, Y_{j}^{(1)}\right)=0, \operatorname{cov}\left(Y_{i}^{(2)}, Y_{j}^{(2)}\right)=0$ respectivamente,

donde $\lambda_{i}$ son los autovalores de la matriz $\Sigma_{2}^{-1} \Sigma_{1}$ donde $\lambda_{1} \geq \lambda_{2} \geq \ldots \geq \lambda_{p}$.

En el contexto descrito, tienen particular importancia las combinaciones lineales con razones o cocientes extremos de varianzas en ambos grupos, es decir:

$$
\vec{Y}^{(1)}=\left[\begin{array}{c}
Y_{\max }^{(1)} \\
Y_{\min }^{(1)}
\end{array}\right]=\left[\begin{array}{l}
{\overrightarrow{\beta_{1}^{\prime}}}^{(1)} \\
{\overrightarrow{\beta_{p}^{\prime}}}^{(1)}
\end{array}\right] \text { y } \vec{Y}^{(1)}=\left[\begin{array}{c}
Y_{\max }^{(2)} \\
Y_{\min }^{(2)}
\end{array}\right]=\left[\begin{array}{c}
{\overrightarrow{\beta_{1}^{\prime}}}^{(2)} \\
{\overrightarrow{\beta_{p}^{\prime}}}^{(2)}
\end{array}\right]
$$

cuyas matrices de covarianza son:

$$
\Sigma_{\vec{Y}^{(1)}}=\left[\begin{array}{ll}
1 & 0 \\
0 & 1
\end{array}\right] \text { y } \Sigma_{\vec{Y}^{(2)}}=\left[\begin{array}{cc}
\lambda_{1} & 0 \\
0 & \lambda_{p}
\end{array}\right] \text { respectivamente }
$$

El resultado es de particular importancia por que tenemos dos combinaciones lineales, las de mayor y menor cociente de varianzas, en cada una de las poblaciones, que pueden usarse para realizar las representaciones gráficas y la consecuente reducción de dimensión 
de las variables, toda vez que las matrices de covarianzas de las poblaciones concurrentes son diferentes.

\section{ESTIMACIÓN DE PARÁMETROS}

Para la presentación de los resultados del ACP y del ACPG se ha admitido que las dos poblaciones tienen parámetros conocidos, por lo que se hace necesario hacer la siguiente consideración.

Para la muestra aleatoria $\vec{x}_{1}, \ldots, \vec{x}_{n}$ obtenida de la población con vector de medias $\overrightarrow{\mu^{\prime}}=\left(u_{1}, \ldots, u_{p}\right)$ y matriz de covarianza $\Sigma, \vec{X}$ y $S$ son los correspondientes estimadores, por lo que para estimar (1) se obtienen los autovalores y autovectores de la matriz de covarianzas de la muestra. Se usan los dos primeros autovectores para estimar las dos primeras componentes principales y sus varianzas serán los eorrespondientes autovalores asociados.

En el caso de dos poblaciones, para las muestras aleatorias $\vec{x}_{1}^{(g)}, \vec{x}_{2}^{(g)}, \ldots, \vec{x}_{n_{g}}^{(g)}, g=1,2$, de tamaños $n_{1}$ y $n_{2}, \overrightarrow{\bar{x}}_{1}, \overrightarrow{\bar{x}}_{2}, S_{1}$ y $S_{2}$ son los correspondientes estimadores de $\vec{\mu}^{(g)}$ y $\Sigma_{g}$ respectivamente. Para estimar (2), (3) y (4) se obtiene los autovalores y autovectores de la matriz $S_{2}^{-1} S_{1}$. Se usan los autovectores asociados al máximo y mínimo autovalores de la matriz $S_{2}^{-1} S_{1}$.

Luego del análisis descriptivo mediante el ACP y ACPG, se realiza el análisis confirmatorio mediante el contraste de la hipótesis para la diferencia de los vectores de medias $\vec{\mu}^{(g)}$ y se responde a la pregunta de si los vectores de medias de las comunidades de Cajatambo y Unchus son o no diferentes. Bajo la hipótesis nula de que los vectores de medias son iguales, $\left(H_{0}: \vec{\mu}^{(1)}=\vec{\mu}^{(2)}\right)$, la estadística $\frac{n_{1}+n_{2}-p-1}{\left(n_{1}+n_{2}-2\right) p} T^{2}$ tiene distribución $F$ con $\left(p, n_{1}+n_{2}-p-1\right)$ grados de libertad, donde $T^{2}=\frac{n_{1} n_{2}}{n_{1}+n_{2}}\left(\vec{x}_{1}-\overrightarrow{\bar{x}}_{2}\right) S^{-1}\left(\overrightarrow{\bar{x}}_{1}-\overrightarrow{\bar{x}}_{2}\right)$ y $S=\frac{\left(n_{1}-1\right) S_{1}+\left(n_{2}-1\right) S_{2}}{n_{1}+n_{2}-2}$ (Jonshon, 2000; Seber, 1984; entre otros). 


\section{RESULTADOS Y DISCUSIÓN}

Para el análisis estadístico mediante CP, se consideró la muestra total de 80 plantas de Minthostachys y en el Laboratorio de Etnobotánica se realizaron las mediciones de las siguientes variables:

X1: Largo del pedicelo de la flor

X2: Ancho del pedicelo de la flor

X3: Número de venas del cáliz tubular

X4: Largo de la corola de la flor

X5: Ancho de la corola de la flor

Con las mediciones obtenidas, se procede a la obtención del vector de medias de dichas variables y la correspondiente matriz de covarianzas. Con los dos primeros autovectores de la matriz de covarianzas se estima (1), es decir se definen las dos primeras Componentes Principales estandarizadas (centradas en cero y de varianza 1),

$$
\begin{aligned}
& Y_{1}=0,632 X_{1}+0,6381 X_{2}+0,4407 X_{3}+0,7580 X_{4}+0,6227 X_{5}-14,5764 \\
& Y_{2}=0,6465 X_{1}-0,0996 X_{2}-1,2574 X_{3}-0,0849 X_{4}+0,1885 X_{5}-11,9988
\end{aligned}
$$

variables con las mayores varianzas, $\lambda_{1}=1,54$ y $\lambda_{2}=0,39$ respectivamente.

Reemplazando las puntuaciones originales de cada individuo en cada una de las combinaciones lineales $Y_{1}$ y $Y_{2}$ se obtiene las puntuaciones en las CP para cada individuo. Dicha información presentada en el Gráfico No 1, indica claramente la presencia de tres especies diferentes de Minthostachys.

Las dos primeras componentes principales retienen el $80.8 \%$ de la variación total del problema original. A partir de la matriz de correlaciones entre las componentes principales y las variables originales se encuentra que la primera componente principal explica el $63,5 \%$ de la variación del largo de la flor, 78,8\% de la variación del ancho de la flor, $74,8 \%$ de la variación del largo de la corola y 79,6\% de la variación del ancho de la corola.; mientras que la segunda componente explica el 73,6\% de la variación del número de venas del cáliz. 


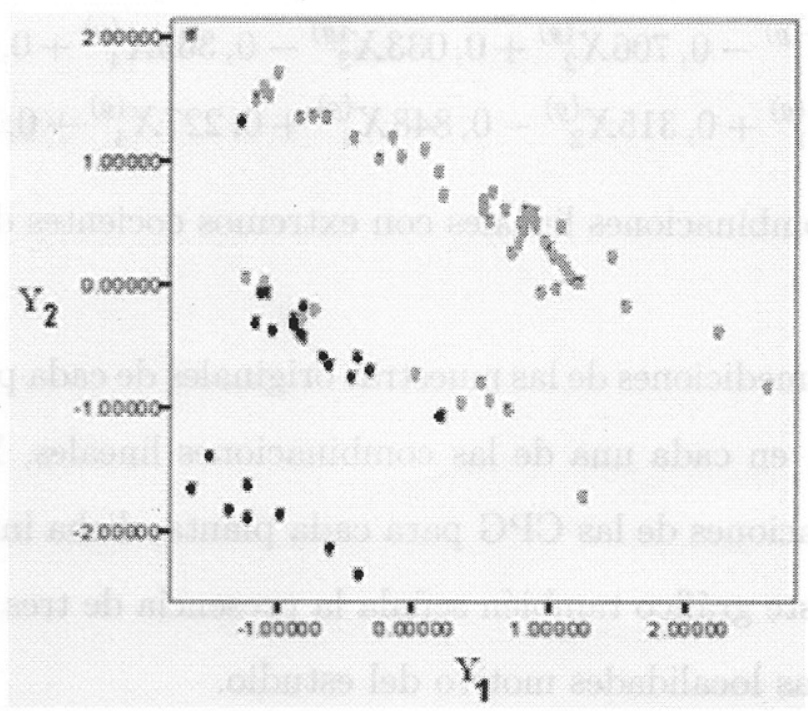

Gráf. 1: Resultado del análisis de componentes principales.

También se realizó el correspondiente gráfico con los dos primeros factores generados luego de aplicar la metodología del Análisis Factorial con el auxilio del software SPSS. La estructura encontrada fue similar al del Gráfico No 1, por dicha razón se ha obviado su presentación.

Mediante el análisis de CPG se logró lo siguiente. De las 80 plantas de Minthostachys, 56 de ellas fueron recolectadas en la localidad de Cajatambo y las 24 restantes en la localidad de Unchus. Con dicha información se estimaron los vectores de medias y las correspondientes matrices de covarianzas para las variables analizadas de ambas localidades. La prueba de hipótesis para comparar las matrices de covarianzas (Mardia, 1979), realizada con el auxilio del SPSS, arrojó matrices de covarianzas diferentes, por lo que pudo continuarse con el análisis de Componentes Principales Generalizados (CPG) y se procedió a estimar las relaciones (2), (3) y (4) respectivamente.

Con los autovectores de la matriz

$$
S_{2}^{-1} S_{1}=\left[\begin{array}{ccccc}
-0.4160 & -0.0694 & 0.0130 & -0.5597 & -0.3587 \\
-0.7058 & -0.0874 & -0.8153 & 0.1778 & 0.3148 \\
0.0327 & 0.0401 & -0.0224 & 0.5400 & -0.8484 \\
-0.3628 & -0.2758 & 0.1934 & 0.5783 & 0.2268 \\
0.4428 & 0.9539 & 0.5452 & -0.1706 & 0.0309
\end{array}\right]
$$

asociados a sus autovalores máximo y mínimo, $\lambda_{1}=6,46$ y $\lambda_{5}=0,40$, se definieron las siguientes $\mathrm{CPG}$ : 


$$
\begin{aligned}
& Y_{\max }^{(g)}=-0,416 X_{1}^{(g)}-0,706 X_{2}^{(g)}+0,033 X_{3}^{(g)}-0,363 X_{4}^{(g)}+0,443 X_{5}^{(g)} \\
& Y_{\min }^{(g)}=-0,359 X_{1}^{(g)}+0,315 X_{2}^{(g)}-0,848 X_{3}^{(g)}+0,227 X_{4}^{(g)}+0,031 X_{5}^{(g)}
\end{aligned} \quad g=1,2
$$

es decir, son las combinaciones lineales con extremos cocientes de varianzas en ambas localidades.

Reemplazando las mediciones de las muestras originales de cada planta de Minthostachys de ambas localidades, en cada una de las combinaciones lineales, $Y_{\max }^{(g)}$ y $Y_{\min }^{(g)}, g=1,2$, se obtienen las puntuaciones de las CPG para cada planta, dicha información se presenta en el Gráfico No 2. Este gráfico también señala la presencia de tres especies diferentes de Minthostachys entre las localidades motivo del estudio.

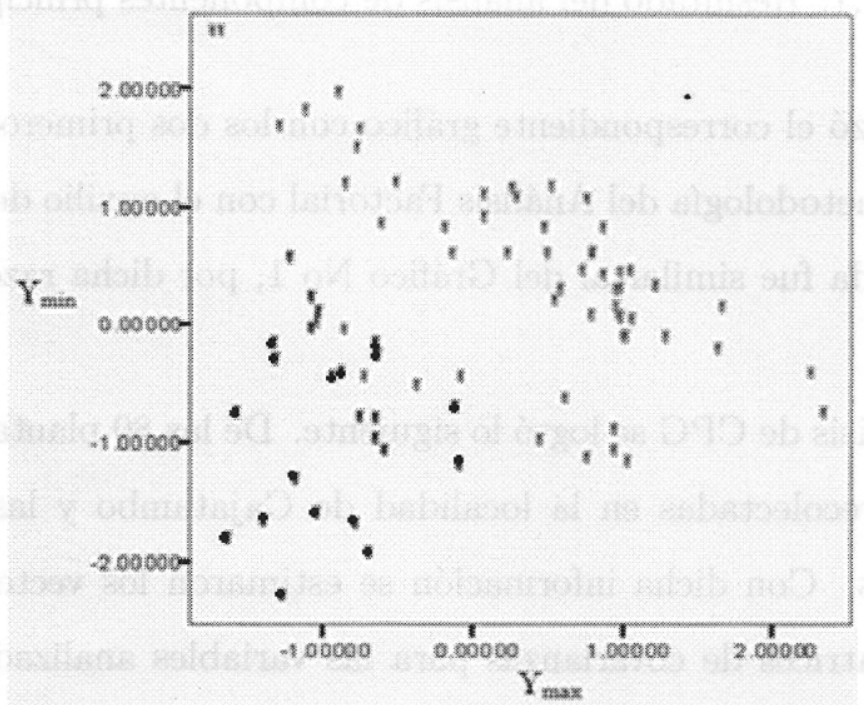

Gráf. 2: Resultado del análisis de componentes principales generalizados.

Los Gráficos No 1 y No 2, indican claramente la presencia de tres especies diferentes de Minthostachys. Una de ellas se encuentra en ambas localidades, además cada localidad tiene una especie que sólo se encuentra en su territorio.

En el Análisis confirmatorio se llegó a lo siguiente. El valor de $T^{2}$ de Hotelling, 203.6, correspondiente a la comparación de los vectores de medias, se transforma a la estadística $F$ y toma el valor de $F_{c}=38.6$, que comparado con el cuantil de la distribución $F$ con 5 y 74 grados de libertad con una probabilidad de $0,95, F(5,74 ; 0,95)=2,34$, resulta evidentemente significativo.

Dicho resultado, al nivel de significación de 0,05, permite concluir, que existe evidencia suficiente para afirmar que los vectores de medias de las dos localidades son diferentes, es decir las especies encontradas en Cajatambo y Unchos son diferentes, a pesar de existir 
una especie común en ambos lugares.

\section{CONCLUSIONES}

El análisis de Componentes Principales, el Análisis Factorial y el análisis de Componentes Principales Generalizados muestran que son tres las especies de Minthostachys encontradas en el Distrito de Cajatambo del Departamento de Lima y en la localidad de Unchus del Departamento de Ancash. Es decir existe una especie de Minthostachys en Cajatambo y otra especie en Unchus; y una tercera especie cuya presencia es común a ambas comunidades.

El análisis de confirmatorio de comparación de los vectores de medias muestra la diferencia significativa entre las especies de Minthostachys de las localidades de Cajatambo y Unchus, a pesar de que una es común en ambas localidades.

\section{FINANCIAMIENTO}

Expresamos nuestro reconocimiento al Consejo Superior de Investigaciones de la UNMSM que financió la ejecución del estudio motivo de la presente publicación. 


\section{REFERENCIAS BIBLIOGRÁFICAS}

[1] Alban, C.J. Un registro de datos etnobotánicos. Boletín de Lima, No 39. (1985).

[2] Brako L. \& J. L. Zarucchi. Catalogue of the Flowering Plants and Gymnosperms of Perú / Catálogo de las Angiospermas y Gimnospermas del Perú. Monogr. Syst. Bot. Missouri Botanical Garden. 1286pp. (1993).

[3] Flury, B. Some relation between the comparison of covariance matrices and principal component analysis Computational Statistics \&6 Data Analysis 1, 97-109. (1983)

[4] Gómez, D. Una aplicación de Discriminación cuadrática en la agricultura. Pesquimat. Revista de Investigación de la Facultad de Ciencias Matemáticas. UNMSM. Vol V No 1. ( 2002).

[5] Gómez, D. Caracterización de patrones de variación morfológica de Minthostachys mediante métodos estadísticos multivariantes. Vol X No 2. (2007).

[6] INEI, Censos Nacionales $X$ de población y $V$ de vivienda. (2005)

[7] Manly, B. Multivariate statistical methods. Third edition Chapman Hall/CRC. (2004)

[8] Mostacero, B. Fredericksen Manual de métodos básicos de muestreo y análisis en ecología vegetal. Bolford. (2000).

[9] Jonson, D.E.Métodos multivariados aplicados al análisis de datos, Thomsom Editores, Méjico. (2000).

[10] Roy, S.N. Some aspects of multivariate analysis. John Wiley, New York. (1957).

[11] Seber, G.A.F. Multivariate observations, John Wiley, New York. . (1984). 